\title{
ACCELERATION DE LA CONVERGENCE DES SUITES DONT LE RAPPORT DES ERREURS EST BORNE
}

\author{
J. P. Delahaye $\left({ }^{1}\right)$
}

\begin{abstract}
The family of sequences with a bounded ratio of errors is studied, with the point of view of convergence acceleration. In the first part of the paper we establish that this family is too big, in the sense: there is no transformation which could accelerate all its sequences. In the second part we study the sub-family of periodically-linear sequences, and we propose a few algorithms accelerating this sub-family, those algorithms are generalizations of the Aitken's process.
\end{abstract}

\section{0 . Introduction.}

Une famille $S$ de suites convergentes étant donnée, le problème d'accélération de la convergence le plus simple qu'on puisse se poser c'est: existe-t-il une transformation qui accélère toute suite de $S$ ?

Deux types de réponses peuvent être donnés:

1) Les réponses positives: on prend une transformation déjà connue (ou ori en construit une nouvelle) et on montre qu'elle accélère toutes les suites de la famille $S$. Ce type de résultats a été énoncé à propos de pratiquement toutes les méthodes d'accélèration existantes ([2] [3] [4] etc...), c'est lui qui les justifie.

2) Les réponses négatives: à l'aide d'un raisonnement par l'absurde et en considérant des types de transformations plus ou moins généraux, on montre l'impossibilité d'une transformation accélérant toutes les suites de $S$. Ces résultats négatifs indiquent, soit qu'il faut envisager une famille de transformations plus grande (certains résultats de Pennacchi [15] et Germain-Bonne [12]

- Received March 5, 1980.

(1) Université des Sciences et Techniques de Lille. Laboratoire d'Informatique B. P. 36 59650 Villeneuve - d'Ascq France. 
doivent s'interpréter ainsi), soit qu'il faut diminuer $S$ et n'essayer d'accélérer que certaines des suites de $S$. (C'est le sens à donner aux résultats de [10]).

C'est grâce à la conjonction de ces deux types de résultats que l'on peut espérer cerner les possibilités de l'accélération de convergence.

Dans ce travail nous donnons des résultats des deux types à propos de la famille des suites $\left(x_{n}\right)$ de nombres réels dont le rapport $\left(x_{n+1}-x\right) /\left(x_{n}-x\right)$ $\left(x=\lim _{n \rightarrow \infty} x_{n}\right)$ ou $\left(x_{n+2}-x_{n+1}\right) /\left(x_{n+1}-x_{n}\right)$ reste borné. Au paragraphe 1 nous montrons qu'il est illusoire de vouloir accélérer toutes ces suites par une même transformation. Au paragraphe 2 nous établissons que certaines sous-classes de cette famille peuvent être accélérées; pour cela nous proposons des procédés nouveaux généralisant en un certain sens le procédé $\Delta^{2}$ d'Aitken [1], dont certains utilisent, pour des calculs auxiliaires, des algorithmes de détermination de la période [7].

1. Problème général de l'accélération des suites telles que: $0 \leq \lambda \leq \Delta x_{n+1} / \Delta x_{n} \leq \mu<1$.

Pour certains algorithmes comme le procédé $\Delta^{2}$ d'Aitken [3] et le procédé d'Overholt [2] [3], une conditoin suffisante assurant que la suite transformée $\left(t_{n}\right)$ a la même limite que la suite donnée $\left(x_{n}\right)$ s'énonce:

$$
\left(x_{n+2}-x_{n+1}\right) /\left(x_{n+1}-x_{n}\right) \notin[\alpha, \beta] \text {, avec } \alpha<1<\beta \text {. }
$$

Cependant on s'aperçoit par le calcul [13] que les suites $\left(a, a b, a^{2} b, a^{2} b^{2}, a^{3} b^{2}, \ldots\right)$ avec $a \neq b$ ne sont accélérées ni par le procédé $\Delta^{2} \mathrm{~d}^{\prime}$ Aitken ni par le procédé $W$. de Lubkin [14], bien que ces suites vérifient la relation ci-dessus.

Nous allons montrer que l'existence de suites vérifiant cette relation, et non accélérées, n'est pas spécifique de ces deux procédés (qui sont en un certain sens les procédés de base de l'accélération non linéaire [5]).

C'est le but du premier théorème où l'on énonce que la connaissance de deux bornes $\lambda, \mu(\lambda<\mu)$ pour le rapport $\left(x_{n+2}-x_{n+1}\right) /\left(x_{n+1}-x_{n}\right)$ n'est suffisante pour aucun procédé. De façon précise:

THÉORÈME 1. Soient $\lambda \in \mathbf{R}, \mu \in \mathbf{R}$ vérifiant: $0 \leq \lambda<\mu<1$.

Il n'existe aucune transformation qui, utilisant seulement $\left(x_{0}, x_{1}, \ldots, x_{n}\right)$ pour le calcul de $t_{n}$, accélère toute suite telle que:

$$
\left(P_{\lambda \mu}\right) \quad \forall n \in \mathrm{N}: \lambda \leq\left(x_{n+2}-x_{n+1}\right) /\left(x_{n+1}-x_{n}\right) \leq \mu
$$


On rappelle que la suite $\left(t_{n}\right)$ est dite accélérer la suite $\left(x_{n}\right)$ convergente vers $x$ si:

$$
\lim _{n \rightarrow \infty} t_{n}=x \text { et } \lim _{n \rightarrow \infty}\left(t_{n}-x\right) /\left(x_{n}-x\right)=0 .
$$

\section{REMAROUES.}

1) Une « transformation utilisant seulement $\left(x_{0}, x_{1}, \ldots, x_{n}\right)$ pour le calcul de $t_{n}$ » est défini par la donnée d'une suite $f_{0}, f_{1}, \ldots, f_{n}, \ldots$ des fonctions de $1,2, \ldots$ $\ldots, n+1, \ldots$ variables; la suite transformée $\left(t_{n}\right)$ étant obtenue par:

$$
t_{0}=f_{0}\left(x_{0}\right), t_{1}=f_{1}\left(x_{0}, x_{1}\right), \ldots, t_{n}=f_{n}\left(x_{0}, x_{1}, \ldots, x_{n}\right), \ldots
$$

Le théorème 1 doit donc se comprendre comme:

il n'existe aucune suite de fonctions $f_{0}, f_{1}, \ldots, f_{n}, \ldots$ telle que toute suite $\left(x_{n}\right)$ satisfaisant $\left(P_{\lambda_{\mu}}\right)$ soit transformée par $(F)$ en une suite accélérant $\left(x_{n}\right)$.

Cette notion de transformation que nous venons de détailler est très générale puisqu'on n'impose aucune condition aux fonctions $f_{0}, f_{1}, \ldots, f_{n}, \ldots$ Elle correspond d'ailleurs à la notion d'algorithme de transformation de suites définie et utilisée dans [9] [10]. en précisant toutefois: $C\left(n, s, s^{\prime}\right)=(0, n)$;

Si par exemple on prend $f_{0}, f_{1}, \ldots, f_{n}, \ldots$ définies par:

$$
\left\{\begin{array}{l}
f_{0}\left(x_{0}\right)=x_{0}, f_{1}\left(x_{0}, x_{1}\right)=x_{1} \\
f_{n}\left(x_{0}, x_{1}, \ldots, x_{n}\right)=x_{n}-\left(x_{n}-x_{n-1}\right)^{2} /\left(x_{n}-2 x_{n-1}+x_{n-2}\right) \text { si } n \geq 2
\end{array}\right.
$$

on obtient un procédé de transformation analogue au procédé d'Aitken [1] dont on peut montrer qu'il accélère toutes les suites $\left(x_{n}\right)$ à convergence linéaire, c'està-dire satisfaisant:

$$
\exists \lambda \in \mathbf{R}, 0<|\lambda|<1 \lim _{n \rightarrow \infty}\left(x_{n+2}-x_{n+1}\right) /\left(x_{n+1}-x_{n}\right)=\lambda
$$

(voir [3] [6] [8] [15]).

2) Le théorème 1 est évidemment valable pour toute famille de suites plus grande que la famille des suites vérifiant $\left(P_{\lambda p}\right)$.

Le lemme suivant dont la démonstration est évidente nous sera utile:

LEMME. Soit $\left(x_{n}\right)$ une suite de nombres réels.

S'il existe $n_{0} \in \mathbf{N}$ et $\lambda \in \mathbf{R}(|\lambda|<1)$ tels que: 


$$
\forall n \geq n_{0}: x_{n+1}-x_{n}=\lambda\left(x_{n}-x_{n-1}\right) .
$$

Alors:
i) $\forall k \in \mathbf{N}: x_{n_{0}+k+1}=x_{n_{0}}+\frac{\lambda\left(1-\lambda^{k+1}\right)}{1-\lambda}\left(x_{n_{0}}-x_{n_{0}-1}\right)$
ii) $\lim _{n \rightarrow \infty} x_{n}=x_{n_{0}}+\frac{\lambda}{1-\lambda}\left(x_{n_{0}}-x_{n_{0}-1}\right)$.

DÉmonstration DU THÉORÈME 1. Supposons donnée une transformation $A$ n'utilisant pour le calcul de $t_{p}$ que les points $\left(x_{0}, x_{1}, \ldots, x_{p}\right)$ et accélérant toute suite vérifiant $\left(P_{\lambda \mu}\right)$.

Nous allons trouver une contradiction. Pour cela nous allons construire des suites $\left(x_{n}{ }^{0}\right),\left(x_{n}{ }^{1}\right),\left(x_{n}{ }^{2}\right), \ldots$ vérifiant $\left(P_{\lambda \mu}\right)$ et donc, accélérées par $A$; ces suites nous permettront d'obtenir une autre suite $\left(x_{n}\right)$ satisfaisant encore $\left(P_{\lambda \mu}\right)$ et dont nous montrerons qu'elle n'est pas accélérée par $A$.

On pose: $\alpha=\frac{\mu}{1-\mu}-\frac{\lambda}{1-\lambda}>0, \beta=\min \left\{\frac{\alpha-\alpha \lambda}{4 \lambda} ; 1\right\}>0$

$$
\delta=\frac{1}{2} \alpha-\frac{\lambda \beta}{1-\lambda}>0, \quad \theta=\frac{\delta}{\lambda /(1-\lambda)+\alpha / 2}>0
$$

Soit $\left(x_{n}^{0}\right)$ la suite définie par:

$$
x_{0}^{0}=0 ; \quad x_{1}^{0}>x_{0}^{0} ; \quad \forall n \in \mathbf{N} \frac{x_{n+2}^{0}-x_{n+1}^{0}}{x_{n+1}^{0}-x_{n}^{0}}=\lambda
$$

A transforme $\left(x_{n}^{0}\right)$ en une suite que nous notons $\left(t_{n}^{0}\right)$ et qui, par hypothèse, accélère $\left(x_{n}^{0}\right)$. Il existe donc $n_{0} \in \mathbf{N}$ tel que:

$$
\left|\frac{t_{n_{0}}^{0}-x_{n_{0}}^{\lambda}}{x_{n_{0}}^{0}-x_{n_{0}}^{\lambda}}\right| \leq \beta
$$

où on a posé: $x_{n_{0}}^{\lambda}=x_{n_{0}}^{0}+\frac{\lambda}{1-\lambda}\left(x_{n_{0}}^{0}-x_{n_{0}-1}^{0}\right)$

(d'après le lemme: $x^{\lambda} n_{0}=\lim _{n \rightarrow \infty} x_{n}^{0}$ ).

Soit $\left(x_{n}^{1}\right)$ la suite définie par: 


$$
\begin{aligned}
& x^{x_{n}^{1}=x_{n}^{0} \text { si } n \leq n_{0}} \\
& \left\{\begin{array}{l}
x_{n}^{1}-x_{n-1}^{1} \\
x_{n-1}^{1}-x_{n-2}^{1}
\end{array}=\mu \text { si } n>n_{0} .\right.
\end{aligned}
$$

D'après le lemme cette suite est croissante et convergente vers:

$$
x_{n_{0}}^{\mu}=x_{n_{v}}^{0}+\frac{\mu}{1-\mu}\left(x_{n_{0}}^{0}-x_{n_{0}-1}^{0}\right)>x_{n_{3}}^{\lambda} .
$$

11 existe donc $n_{1} \in \mathbf{N}, n_{1}>n_{0}$, vérifiant:

$$
x_{n_{1}}^{1} \geq x^{\lambda \mu}{ }_{n}
$$

où on a posé:

$$
x^{\lambda \mu}{ }_{n_{0}}=\left(x_{n_{0}}^{\mu}+x_{n_{0}}^{\lambda}\right) / 2=x_{n_{0}}^{\lambda}+\alpha\left(x_{n_{3}}^{0}-x_{n_{0}-1}^{0}\right) / 2 .
$$

On définit alors $\left(x_{n}^{2}\right)$ par:

$$
\left\{\begin{array}{l}
x_{n}^{2}=x_{n}^{1} \text { si } n \leq n_{1} \\
\frac{x_{n}^{2}-x_{n-1}^{2}}{x_{n-1}^{2}-x_{n-2}^{2}}=\lambda \text { si } n>n_{1} .
\end{array}\right.
$$

A transforme $\left(x_{n}^{2}\right)$ en une suite que nous notons $\left(t_{n}^{2}\right)$ et qui, par hypothèse, accélère $\left(x_{n}^{2}\right)$; il existe donc $n_{2} \in \mathbf{N}, n_{2}>n_{1}$, tel que:

$$
\left|\frac{t_{n_{2}}^{2}-x_{n_{3}}^{\lambda}}{x_{n_{3}}^{2}-x_{n_{3}}^{\lambda}}\right| \leq \beta
$$

où on a posé $x_{n_{2}}^{\lambda}=x_{n_{3}}^{2}+\frac{\lambda}{1-\lambda}\left(x_{n_{2}}^{2}-x_{n_{2}-1}^{2}\right)$

(d'après le lemme: $x_{n_{2}}^{\lambda}=\lim _{n \rightarrow \infty} x_{n}^{2}$ ).

On continue ainsi, en suivant le même principe, ce qui nous donne des suites que nous notons $\left(x_{n}^{3}\right),\left(x_{n}^{4}\right), \ldots$

On considère alors la suite:

$$
\left(x_{n}\right)=\left(x_{0}^{0}, x_{1}^{0}, \ldots, x_{n_{0}}^{0}, x_{n_{0}+1}^{1}, x_{n_{0}+2}^{1}, \ldots, x_{n_{1}}^{1}, x_{n_{1}+1}^{2}, x_{n_{1}+2}^{2}, \ldots\right) .
$$


Cette suite est convergente car:

$$
\forall n \in \mathrm{N}: \frac{x_{n+2}-x_{n+1}}{x_{n+1}-x_{n}} \in\{\lambda, \mu\} .
$$

La limite $x$ de cette suite vérifie:

$$
x \geq x_{n_{0}}^{\lambda \mu}, x \geq x^{\lambda \mu}{ }_{\mu_{2}}, \ldots
$$

On a: $t_{n_{0}}^{0} \geq x_{n_{0}}^{0}$ (resp. $t_{n_{2}}^{2} \geq x_{n_{9}}^{2}, \ldots$ ) car:

$$
\begin{aligned}
t_{n_{0}}^{0} & =x_{n_{0}}^{0}+x_{n_{0}}^{\lambda}-x_{n_{0}}^{0}+t_{n_{0}}^{0}-x_{n_{0}}^{\lambda} \geq \\
& \geq x_{n_{0}}^{0}+\left(x_{n_{0}}^{\lambda}-x_{n_{0}}^{0}\right)-\left|t_{n_{0}}^{0}-x_{n_{j}}^{\lambda}\right| \geq \\
& \geq x_{n_{0}}^{0}+\left(x_{n_{3}}^{\lambda}-x_{n_{0}}^{0}\right)-\beta\left|x_{n_{0}}^{0}-x_{n_{0}}^{\lambda}\right|= \\
& =x_{n_{0}}^{0}+(1-\beta)\left(x_{n_{0}}^{\lambda}-x_{n_{0}}^{0}\right) \geq x_{n_{0}}^{0} .
\end{aligned}
$$

La fonction homographique $z \rightarrow\left(z-t^{0} n_{0}\right) /\left(z-x_{n_{0}}^{0}\right)$ est donc croissante; donc:

$$
\left(x-t_{n_{n_{j}}}^{0}\right) /\left(x-x_{n_{j}}^{0}\right) \geq\left(x_{n_{0}}^{\lambda \mu}-t_{n_{0}}^{0}\right) /\left(x^{\lambda \mu}{ }_{n_{j}}-x_{n_{0}}^{0}\right) \text {. }
$$

Comme la transformation $A$ n'utilise pour le calcul de $t_{p}$ que les points $\left(x_{0}, x_{1}, \ldots, x_{p}\right)$ et que la suite $\left(x_{n}\right)$ est identique à la suite $\left(x_{n}^{0}\right)$ pour $n \leq n_{0}$ il en résulte (en notant $\left(t_{n}\right)$ la suite transformée de $\left(x_{n}\right)$ par $A$ ) que $t_{n_{0}}=t^{0}{ }_{n_{j}}$; donc:

(*)

$$
\left(x-t_{n_{0}}\right) /\left(x-x_{n_{0}}\right) \geq\left(x^{\lambda \mu}{ }_{n_{0}}-t_{n_{0}}^{0}\right) /\left(x^{\lambda \mu}{ }_{n_{0}}-x_{n_{0}}^{0}\right) .
$$

D'autre part:

$$
\begin{aligned}
x^{\lambda \mu}{ }_{n_{0}}-t_{n_{0}}^{0} & =x^{\lambda \mu_{n_{0}}}-x_{n_{j}}^{\lambda}+x_{n_{0}}^{\lambda}-t_{n_{1}}^{0} \geq \\
& \geq \alpha\left(x_{n_{0}}^{0}-x_{n_{0}-1}^{0}\right) / 2-\left|x_{n_{0}}^{\lambda}-t_{n_{0}}^{0}\right| \geq \\
& \geq \alpha\left(x_{n_{0}}^{0}-x_{n_{0}-1}^{0}\right) / 2-\beta\left|x_{n_{0}}^{\lambda}-x_{n_{1}}^{\lambda}\right|= \\
& =\delta\left(x_{n_{j}}^{0}-x_{n_{0}-1}^{0}\right)
\end{aligned}
$$

donc:

$$
\frac{x_{n_{0}}^{\lambda \mu_{0}}-t_{n_{3}}^{0}}{x_{n_{0}}^{\lambda \mu_{0}}-x_{n_{3}}^{0}} \geq \frac{\delta\left(x_{n_{3}}^{0}-x_{n_{1}-1}^{0}\right)}{x_{n_{0}}^{\lambda \mu_{0}}-x_{n_{j}}^{0}}=
$$




$$
=\frac{\delta\left(x_{n_{0}}^{0}-x_{n_{0}-1}^{0}\right)}{x_{n_{0}}^{\lambda}-x_{n_{0}}^{0}+\alpha\left(x_{n_{0}}^{0}-x_{n_{0}-1}^{0}\right) / 2}=\theta \text {. }
$$

Cette inégalité avec l’inégalité (*) donne:

$$
\left(x-t_{n_{0}}\right) /\left(x-x_{n ;}\right) \geq \theta
$$

De la même façon on obtient:

$$
\begin{aligned}
& \left(x-t_{n_{1}}\right) /\left(x-x_{n_{2}}\right) \geq \theta \\
& \left(x-t_{n_{4}}\right) /\left(x-x_{n_{4}}\right) \geq \theta \ldots
\end{aligned}
$$

Ce qui montre que $\left(t_{n}\right)$ n'accélère pas $\left(x_{n}\right)$.

REMARQue 3. On a seulement utilisé des suites vérifiant:

$\left(P_{\lambda \mu}^{\prime}\right) \quad \forall n \in \mathbf{N}:\left(x_{n+2}-x_{n+1}\right) /\left(x_{n+1}-x_{n}\right) \in\{\lambda, \mu\}$.

On peut donc dire que le théorème 1 est frai pour la famille des suites vérifiant $\left(P_{\lambda \mu}^{\prime}\right)$.

Corollaire 1. Soient $\lambda \in \mathbf{R}, \mu \in \mathbf{R}$ vérifiant: $0 \leq \lambda<\mu<1$.

Ii n'existe aucune transformation qui utilisant seulement $\left(x_{0}, x_{1}, \ldots, x_{n}\right)$ pour le calcul de $t_{n}$ accélère toute suite telle que:

$\left(P^{\prime \prime} \lambda_{\mu}\right) \quad \forall n \in \mathbf{N}: \lambda \leq\left(x_{n+1}-x\right) /\left(x_{n}-x\right) \leq \mu$

où:

$$
x=\lim _{n \rightarrow \infty} x_{n}
$$

DÉmonstration. Toutes les suites utilisées dans la démonstration précédente vérifient $\left(P_{\lambda \mu}^{\prime \prime}\right)$, car pour chacune d'entre elles:

$$
\lambda=\frac{x_{n}-x_{n}^{\lambda}}{x_{n-1}-x_{n}^{\lambda}} \leq \frac{x_{n}-x}{x_{n-1}-x} \leq \frac{x_{n}-x_{n}^{\mu}}{x_{n-1}-x_{n}^{\mu}}=\mu
$$

où:

$$
\begin{gathered}
x=\lim _{u \rightarrow \infty} x_{n}, x_{n}^{\lambda}=x_{n}+\frac{\lambda}{1-\lambda}\left(x_{n}-x_{n-1}\right) \\
x_{n}^{\mu}=x_{n}+\frac{\mu}{1-\mu}\left(x_{n}-x_{n-1}\right) .
\end{gathered}
$$


Remarque 4. Il est possible d'établir que pour tout $\lambda, \mu(0 \leq \lambda \leq \mu<1)$ :

i) Si $\left(P_{\lambda_{\mu}}\right)$ est vérifié alors $\left(P^{\prime \prime}{ }_{\lambda^{\prime} \mu^{\prime}}\right)$ est vérifié avec:

$$
\lambda^{\prime}=\frac{\lambda \mu-\lambda}{\lambda-1}, \mu^{\prime}=\frac{\lambda \mu-\mu}{\mu-1}
$$

ii) Si $\left(P_{\lambda \mu}^{\prime \prime}\right)$ est vérifié alors $\left(P_{\lambda^{\prime} \mu^{\prime}}\right)$ est vérifié avec:

$$
\lambda^{\prime}=\frac{\lambda \mu-\lambda}{\lambda-1}, \quad \mu^{\prime}=\frac{\lambda \mu-\mu}{\mu-1} .
$$

Corollaire 2. Soit $k \in \mathbf{N}$; soient $\lambda \in \mathbf{R}, \mu \in \mathbf{R}$ vérifiant: $0 \leq \lambda<\mu<1$. I1 n'existe aucune transformation qui, utilisant seulement $\left(x_{0}, x_{1}, \ldots, x_{n}, x_{n+1}, \ldots, x_{n+k}\right)$ pour le calcul de $t_{n}$, accélère toute suite satisfaisant $\left(P_{\lambda \mu}\right)$ (resp. $\left(P_{\lambda \mu}^{\prime \prime}\right)$ ).

DÉmonstration. Il suffit d'établir le résultat pour $\lambda>0$.

Supposons qu'une telle transformation $T$ existe. On définit alors la transformation $T^{\prime}$ suivante:

- aux étapes $i \in\{0,1, \ldots, k-1\} ; t^{\prime}{ }_{i}=x_{i}$.

$\left(\left(t_{n}^{\prime}\right),\left(t_{n}\right)\right.$ désignent respectivement les suites transformées de la suite $\left(x_{n}\right)$ par $T$ et $\left.T^{\prime}\right)$.

- pour $i \in\{k, k+1, \ldots\}: t^{\prime}{ }_{i}=t_{i-k}$.

La transformation $T^{\prime}$ n'utilise pour le calcul de $t^{\prime}{ }_{n}$ que $\left(x_{0}, x_{1}, \ldots, x_{n}\right)$ et:

$$
\lim _{n \rightarrow \infty} \frac{t_{n}^{\prime}-x}{x_{n}-x}=\lim _{n \rightarrow \infty} \frac{t_{n-k}-x}{x_{n}-x}=\lim _{n \rightarrow \infty} \frac{t_{n-k}-x}{x_{n-k}-x}=0
$$

La transformation $T^{\prime}$ contredit donc le théorème 1 (resp. le corollaire 1).

Remaroue 5. On ne peut cependant pas aller plus loin que le corollaire 2, et obtenir des résultats négatifs analogues à ceux du théorème 1 et du corollaire 1 sans limiter le nombre de points utilisés par la transformation pour le calcul de $t_{n}$. En effet si $\left(x_{n}\right)$ est une suite vérifiant $\left(P_{\lambda \mu}\right)$ avec $0<\lambda<\mu<1$ (resp. $0<\lambda<\mu<1$ et $\mu^{2}<\lambda$ ) on peut établir que:

$$
\lim _{n \rightarrow \infty} \frac{x_{n^{2}}-x}{x_{n}-x}=0\left(\text { resp. } \lim _{n \rightarrow \infty} \frac{x_{2 n}-x}{x_{n}-x}=0\right)
$$


ce qui signifie que la transformation définie par $t_{n}=x_{n^{2}}$ (resp. $t_{n}=x_{2 n}$ ) accélère $\left(x_{n}\right)$ (de telles transformations ne présentent évidemment aucun intérêt pratique!).

Des résultats sans limitation sur le nombre de points utilisés pour le calcul de $t_{n}$ ont été obtenus à propos, par exemple, de la classe des suites à convergence logarithmique $([10])$.

Remarque 6 [13]. La classe des suites obtenues par une itération: $x_{n+1}=$ $=f\left(x_{n}\right)$ où $f$ est une fonction lispchitzienne de rapport $\mu<1$ ( $\mu$ fixé) contient toutes les suites utilisées dans la démonstration du théorème 1 . Les résultats négatifs énoncés valent donc pour cette classe de suites.

\section{Accélération des suites à convergence périodico-linéaire.}

Toutes les suites vérifiant $\left(P_{\lambda \mu}\right),\left(P_{\lambda \mu}^{\prime}\right)$ ou $\left(P^{\prime \prime}{ }_{\lambda \mu}\right)$ ne peuvent être accélérées simultanément; cependant toutes les suites à convergence linéaire le sont comme nous l'avons déjà rappelé (remarque 1 ) par le procédé $\Delta^{2}$ d'Aitken (ou d'ailleurs par un procédé du à Germain-Bonne [12]).

Dans ce paragraphe nous généralisons la notion de suite à convergence linéaire et nous proposons des méthodes généralisant en un certain sens le procédé $\Delta^{2}$, et accélérant ces suites.

DÉFInItIon. On dit que la suite $\left(x_{n}\right)$ de nombres reels est de type périodicolinéaire s'il existe $k \in \mathbf{N}^{*}$ tel que:

$$
\left\{\begin{array}{l}
\exists x \in \mathbf{R}, \exists\left(\beta^{0}, \beta^{1}, \ldots, \beta^{k-1}\right) \in \mathbf{R}^{k} ; \beta^{0}, \beta^{1}, \ldots, \beta^{k-1} \notin\{0,1\} \\
\lim _{n \rightarrow \infty} \frac{x_{n k+1}-x}{x_{n k}-x}=\beta^{0}, \lim _{n \rightarrow \infty} \frac{x_{n k+1}-x}{x_{n k+2}-x}=\beta^{1}, \ldots, \lim _{n \rightarrow \infty} \frac{x_{n k+k}-x}{x_{n k+k-1}-x}=\beta^{k-1} .
\end{array}\right.
$$

Le pius petit entier $k \in \mathbf{N}^{*}$ satisfaisant cette relation est appelé période de la suite, et les nombres $\beta^{0}, \beta^{1}, \ldots, \beta^{k-1}$ sont appelés les rapports de la suite.

\section{ReMARQues.}

7) Avec le vocabulaire de [9] dire que $\left(x_{n}\right)$ est de type périodico-linéaire de période $k$ est équivalent à dire que la suite $\left(\left(x_{n+1}-x\right) /\left(x_{n}-x\right)\right)$ est pseudopériodique de période $k$.

8) Une suite de type périodico-linéaire de période $k=1$ est une suite de type linéaire. Si $\left|\beta^{0}\right|<1$, elle converge vers $x$ et c'est une suite à convergence linéaire. La notion de suite de type périodico-linéaire est donc une généralisation de la notion de suite à convergence linéaire. 
Proposition 1. Soit $\left(x_{n}\right)$ une suite de type périodico-linéaire de période $k$ et de rapports $\beta^{0}, \beta^{1}, \ldots, \beta^{k-1}$.

Si $\left|\beta^{0} \beta^{1} \ldots \beta^{k-1}\right|<1$ alors $\left(x_{n}\right)$ est convergente (on dira qu'elle est à convergence périodico-linéaire).

Si $\left|\beta^{0} \beta^{1} \ldots \beta^{k-1}\right|>1$ alors: $\lim _{n \rightarrow \infty} x_{n}=+\infty$.

DÉmonstration. Si $\left|\beta^{0} \beta^{1} \ldots \beta^{k-1}\right|>1$ alors tout $i \in\{0,1, \ldots, k-1\}$ :

$$
\lim _{n \rightarrow \infty} \frac{x_{n k+k+i}-x}{x_{n k+i}-x}=\beta^{0} \beta^{1} \ldots \beta^{k-1}
$$

donc: $\lim _{n \rightarrow \infty}\left|x_{n k+i}-x\right|=+\infty$; donc $\lim _{n \rightarrow \infty}\left|x_{n k+i}\right|=+\infty$;

donc: $\lim _{n \rightarrow \infty}\left|x_{n}\right|=+\infty$.

De même si $\left|\beta^{0} \beta^{1} \ldots \beta^{k-1}\right|<1$ alors pour tout $i \in\{0,1, \ldots, k-1\}$ la suite $\left(x_{n k+i}\right)_{n \in \mathbf{N}}$ est à convergence linéaire de rapport $\beta=\beta^{0} \beta^{1} \ldots \beta^{k-1}$, et de limite $x$; la suite $\left(x_{n}\right)$ est donc convergente vers $x$.

Proposition 2. Soit $\left(x_{n}\right)$ une suite de type périodico-linéaire de période $k$ et de rapports $\beta^{0}, \beta^{1}, \ldots, \beta^{k-1}$ alors:

$$
\text { i) } \begin{gathered}
\lim _{n \rightarrow \infty} \frac{x_{n k+2}-x_{n k+1}}{x_{n k+1}-x_{n k}}=\beta^{0} \frac{\beta^{1}-1}{\beta^{0}-1}, \lim _{n \rightarrow \infty} \frac{x_{n k+3}-x_{n k+2}}{x_{n k+2}-x_{n k+1}}=\beta^{1} \frac{\beta^{2}-1}{\beta^{1}-1}, \ldots, \\
\lim _{n \rightarrow \infty} \frac{x_{n k+k+1}-x_{n k+k}}{x_{n k+k}-x_{n k+k-1}}=\beta^{k-1} \frac{\beta^{0}-1}{\beta^{k-1}-1}
\end{gathered}
$$

ii) $\left|\beta^{0} \beta^{1} \ldots \beta^{k-1}\right| \neq 1=>\lim _{n \rightarrow \infty} \frac{x_{n+2 k}-x_{n+k}}{x_{n+k}-x_{n}}=\beta^{0} \beta^{1} \ldots \beta^{k-1}=\lim _{n \rightarrow \infty} \frac{x_{n+k}-x}{x_{n}-x}$

(voir la démonstration dans $[6]$ ).

Remarque 9. Une sorte de réciproque se trouve énoncée dans [8].

Proposition 3. Soit $k \in \mathbf{N}^{*}$ fixé. Le procédé de transformation de suites défini par:

$$
t_{n}=x_{n}-\frac{\left(x_{n}-x_{n-k}\right)^{2}}{x_{n}-2 x_{n-k}+x_{n-2 k}} \quad n \geq 2 k
$$

accélère la convergence de toute suite à convergence périodico-linéaire de période $k$, et donne la limite exacte (pour tout $n \geq 2 k: t_{n}=x$ ) de toute suite de la forme; 


$$
x_{n}=x+a \prod_{i=0}^{n} \beta_{i}\left(\beta_{i} \text { suite périodique de période } k: \beta_{i+k}=\beta_{i}\right)
$$

\section{DÉMONSTRATYON:}

$$
\frac{t_{n}-x}{x_{n}-x}=1+\left(\frac{x_{n-k}-x}{x_{n}-x}-1\right) \frac{1}{1-\frac{x_{n-k}-x_{n-2 k}}{x_{n}-x_{n-k}}}
$$

ce qui d'après la proposition 2 donne:

$$
\lim _{n \rightarrow \infty} \frac{t_{n}-x}{x_{n}-x}=0
$$

Si $x_{n}=x+a \prod_{i=0}^{n} \beta_{i}$ avec $\beta_{i}$ périodique de période $k$; on $\mathrm{a}$;

$$
t_{n}=x+\left(a \prod_{i=1}^{n \cdots 2 k} \beta_{i}\right) \beta^{2}-\left(a \prod_{i=0}^{\prime \prime-2 \cdot k} \beta_{i}\right) \frac{\left(\beta^{2}-\beta\right)^{2}}{\beta^{2}-2 \beta+1}=x
$$

où on a posé $\beta=\beta_{i} \beta_{i+1} \ldots \beta_{i+k-1}$ (ne dépend pas de $i$ ).

Remarque 10 . Les procédés $\Delta^{2}{ }_{k}$ qui généralisent en un certain sens le procédé $\Delta^{2}$ d'Aitken ne sont eux-même que des cas particuliers des transformations définies par:

$$
t_{n}=x_{n}-\left(x_{n+p+r}-x_{n}\right) /\left(\left(x_{n+2 p+r+q}-x_{n+p+r+q}\right) /\left(x_{n+p+q}-x_{n+q}\right)-1\right)
$$

où $p, q, r$ sont trois paramètres dans $Z$ avec $p+r \neq 0$.

Pour $p=1, r=q=0$ on obtient le procédé $\Delta^{2}$ d'Aitken, pour $p=-k$, $r=q=0$ on obtient le procédé $\Delta^{2}{ }_{k}$ décrit ci-dessus. D'une manère générale et quelles que soient les valeurs prises par $p, q, r,\left(t_{n}\right)$ accélère toute suite $\left(x_{n}\right)$ à convergence linéaire.

Les procédés $\Delta^{2}{ }_{k}$ sont difficiles à utiliser en pratique car leur mise en oeuvre nécessite la connaissance de $k$. Les procédés de transformation de suites que nous allons proposer maintenant ne nécessiteront pas cette connaissance; ils vont être obtenus par combinaison des $\Delta^{2}{ }_{k}$ et d'algorithmes de détermination de la période [7]. Rappelons briévement ce qu'est un algorithme de détermination de la période:

C'es un procédé de calcul, $K$, qui n'utilisant à l'étape $p$ qu'un nombre fini de points de la suite traitée (nous supposerons toujours ici qu'il s'agit des points $\left(x_{0}, x_{1}, \ldots, x_{p}\right)$ fournit une réponse $k(p) \in \mathbf{N}, 0 \leq k(p) \leq p$. 
On dit que $K$ est satisfaisant pour la classe $S$ ( $S$ étant une sous-classe de la classe des suites pseudo-périodiques) si pour tout $\left(x_{n}\right) \in S$, il existe $p_{0} \in \mathbf{N}$ tel que pour $p \geq p_{0}, k(p)$ soit la période de $\left(x_{n}\right)$. L'algorithme I3 $(\rho)\left(\rho \in \mathbf{R}^{+*}\right.$ fixé) présenté dans [7] est satisfaisant pour la classe des suites pseudo-périodiques $\left(x_{n}\right)$ dont les points d'accumulation sont mutuellement distants de plus de $\rho$.

Algorithme $\Delta \mathrm{I} 3(\rho)$.

ETAPE $p(p \geq 2)$. L'étape $p$ de l'algorithme I3 ( $\rho)$ est appliquée à la suite

$$
y_{n}=\frac{x_{n}-x_{n-1}}{x_{n-1}-x_{n-2}} \quad n \in\{2,3, \ldots, p\}
$$

et donne la réponse $k(p)$. On prend alors:

$$
t_{p}=x_{p}-\left(x_{p}-x_{p-k(p)}\right)^{2} /\left(x_{p}-2 x_{p-k(p)}+x_{n-2 k(p)}\right) .
$$

Notations. Soient $\rho \in \mathbf{R}^{+*}, k \in \mathbf{N}^{*}$; on note $P_{\rho, k}$ l'ensemble des suites $\left(x_{n}\right)$ à convergence périodico-linéaire de période $k$, dont les rapports $\beta^{0}, \beta^{1}, \ldots, \beta^{k-1}$ vérifient que

$$
\beta^{0} \frac{\beta^{1}-1}{\beta^{0}-1}, \beta^{1} \frac{\beta^{2}-1}{\beta^{1}-1}, \ldots, \beta^{k-1} \frac{\beta^{0}-1}{\beta^{k-1}-1}
$$

sont mutuellement distants de plus de $\rho$.

Pour $k=1, P_{\rho, k}$ est l'ensemble des suites à convergence linéaire. On pose:

$$
P_{\rho}=\bigcup_{k \in \mathbb{N}^{*}} P_{\rho, k} .
$$

Les inclusions suivantes sont évidentes:

$$
\rho<\rho^{\prime}=>\left(P_{\rho^{\prime}, k} \subset P_{\rho, k}, P_{\rho^{\prime}} \subset P_{\rho}\right) .
$$

THÉORÈME 2.

i) Pour toute suite $\left(x_{n}\right) \in P_{\rho}$ l'algorithme $\Delta \mathrm{I} 3(\rho)$ donne une suite $\left(t_{n}\right)$ qui accélère la convergence de $\left(x_{n}\right)$

ii) Pour toute suite $\left(x_{n}\right) \in P_{p}$ de la forme

$$
x_{n}=x^{*}+a \prod_{i=0}^{n} \beta_{i} \text { avec } \beta_{i} \text { périodique. }
$$


la suite $\left(t_{n}\right)$ donnée par $\Delta \mathrm{I} 3(\rho)$ vérifie:

$$
\exists p_{0} \in \mathbf{N}, \quad \forall p \geq p_{0}: t_{n}=x^{*} .
$$

DÉmONSTRATion.

i) Si $\left(x_{n}\right) \in P_{\rho}$, il existe $p_{0} \in \mathbf{N}$ tel que pour $p \geq p_{0}, k(p)$ soit la période de $\left(x_{n}\right)$ et donc d'après la proposition $3,\left(t_{n}\right)$ accélère la convergence de $\left(x_{n}\right)$.

ii) De la même manière à partir de la proposition 3 .

Remaroue 11. Appliqué à une suite à convergence linéaire, l'algorithme $\Delta \mathrm{I} 3(\rho)$ (quel que soit $\rho \in \mathbf{R}^{*+}$ ) finira par se comporter comme le procédé d'Aitken. On n'aura donc pas spécialement intérêt à l'utiliser pour une suite à convergence linéaire. Par contre, face à une suite périodico-linéaire (par exemple de période 2) alors que le procédé d'Aitken n'accélère pas, l'algorithme $\Delta \mathrm{I} 3(\rho)$ accélère la convergence.

Remaroue 12. Sur le même modèle et en utilisant les divers algorithmes de détermination de la période $\mathrm{I} 1, \mathrm{I} 2, \mathrm{I} 3, \mathrm{I} 4, \mathrm{~J} 1, \mathrm{~J} 2, \mathrm{~J}^{\prime} 1, \mathrm{~J}$ 2 [7], on peut définir d'autres algorithmes d'accélération; chacun d'eux satisfaisant pour des classes spécifiques de suits à convergence périodico-linéaire. (I4 et J1 particulièrement, sont susceptibles de donner des résultats intéressants).

Remarque 13. A la vue des inclusions entre $P_{\rho}$ et $P_{\rho^{\prime}}$ on peut être tenté de fixer une très petite valeur à la constante $\rho$. Cepcadant une telle attitude n'est pas conseillée car plus $\rho$ est petit, plus $p_{0}$ risque d'être grand. Aussi a-t-on intérêt à choisir: $1 / 100 \leq p \leq 1 / 10$, en se réservant de le prendre plus petit seulement si on dispose de becaucoup de points de $\left(x_{n}\right)$.

Remaroue 14. Certaines suites très particulières à convergence périodicolinéaire sont accélérées par l'z-algorithme; de façon précise on montre [11] que les suites de la forme $S+a_{n} \lambda^{n}$ (où $|\lambda|<1, a_{n}$ est $k$-périodique) sont accélérées par la $2 k$-ième colonne de l' $\varepsilon$-algorithme. 


\section{REFERENCES}

[1] A. C. Artken, On Bernouilli's numerical solution of algebraic equations, Proc. Roy. Soc. Edinburgh, 46, (1926), 289-305.

[2] C. Brezinski, Conditions d'application et de convergence de procédés d'extrapolation, Numer. Math. 20, (1972), 64-79.

[3] C. BREZinski, Accélération de la convergence en analyse numérique, Lectures Notes in Mathematics $\mathrm{n}^{0}$ 584, (1977), Springer-Verlag, Heidelberg.

[4] C. Brezinski, Algorithmes d'accélération de la convergence. Etude numérique, (1978), Technip, Paris.

[5] F. CoRdellier, Sur la régularité des procédés $\Delta^{2}$ d'Aitken et $W$. de Lubkin, Pub. A. N. O. $\mathrm{n}^{0} 10$, Université des Sciences et Techniques de Lille. Juin 1979.

[6] J. P. Delahaye, Liens entre la suite du rapport des erreurs et celle du rapport des différences: démonstrations. Pub. A. N. O. $\mathrm{n}^{0} 14$ Université des Sciences et Techniques de Lille Nov. 1979.

[7] J. P. Delahaye, Détermination de la période d'une suite pseudo-périodique, Bulletin de la direction des études et recherches de l'E. D. F., C n 101 (1980), 65-79.

[8] J. P. Delahaye, Liens entre la suite du rapport des erreurs et celle du rapport des différences, C. R. Acad. Sc. Paris A (1980), 343-346.

[9] J. P. Delahaye, Algorithmes pour suites non convergentes, Numerische Mathematik. 333-347.

[10] J. P. Delahaye, B. Germain-Bonne, Quelques résultats négatifs en accélération de la convergence, Numerische Mathematik (1980), 443-457.

[11] A. Genz, Applications of the $\varepsilon^{-a l g o r i t h m}$ to quadrature problems, in "Padé approximants an their applications ", (1973), P. R. Graves-Morris ed. Academic Press, New-York.

[12] B. Germain-Bonne, Estimation de la limite de suites et formalisation de procédés d'accélération de la convergence, Thèse, Université de Lille, (1978).

[13] B. Germain-Bonne, Communication personnelle.

[14] S. Lubkin, A method for summing infinite series, J. Rés. NBS 48 (1952), 228-254.

[15] R. PenNacchI, Le transtormazioni rationali di una successione, Calcolo, 5 (1968), 37-50. 